\title{
La recherche en anglais scientifique et la revue ASp : retour sur vingt ans de publications (1993-2012)
}

\section{Hélène Laffont et Jean-Louis Trouillon}

\section{(2) OpenEdition}

1 Journals

Édition électronique

URL : http://journals.openedition.org/asp/3331

DOI : 10.4000/asp.3331

ISSN : 2108-6354

Éditeur

Groupe d'étude et de recherche en anglais de spécialité

\section{Édition imprimée}

Date de publication : 1 mars 2013

Pagination : 113-158

ISSN : 1246-8185

\section{Référence électronique}

Hélène Laffont et Jean-Louis Trouillon, «La recherche en anglais scientifique et la revue ASp : retour sur vingt ans de publications (1993-2012) », ASp [En ligne], 63 | 2013, mis en ligne le 07 octobre 2014, consulté le 02 novembre 2020. URL : http://journals.openedition.org/asp/3331 ; DOI : https://doi.org/ 10.4000/asp.3331

Ce document a été généré automatiquement le 2 novembre 2020.

Tous droits réservés 


\title{
La recherche en anglais scientifique et la revue $A S p$ : retour sur vingt ans de publications (1993-2012)
}

\author{
Hélène Laffont et Jean-Louis Trouillon
}

\section{Introduction}

1 La présente étude s'intéresse à la revue $A S p^{1}$, revue du GERAS (Groupe d'étude et de recherche en anglais de spécialité), dans laquelle publient un grand nombre de chercheurs en anglais de spécialité. Elle analyse les publications de ces chercheurs de façon à vérifier l'hypothèse selon laquelle le développement de l'anglais de spécialité en tant qu'objet de recherche a été influencé par «les transformations qui marquent notre ère et notre environnement ", comme le posait le texte de cadrage du colloque du GERAS en $2012^{2}$.

Elle s'inscrit dans le prolongement des travaux de Monique Mémet et Michel Van der Yeught (2008) qui proposent un historique de la revue ASp, créée en 1993. Ils indiquent comment les articles publiés peuvent être classés, d'une part en fonction des domaines de spécialité que les chercheurs abordent, d'autre part selon l'approche retenue. Ils notent en premier lieu combien les domaines de spécialité sont multiples: ils en dénombrent huit qui représentent chacun entre 2 et $12 \%$ de l'ensemble (commerce, droit, finance, publicité et marketing, économie, médecine, informatique et technologie, industries sciences et techniques), ainsi que cinq autres domaines peu représentés puisqu'ils ne font l'objet que d'un à cinq articles (militaire, cinéma, administration, art et géologie). Ils remarquent également que la moitié environ (49\%) des articles traite de plusieurs domaines ou n'aborde pas un domaine de spécialité précis. Ils analysent les approches retenues par les auteurs et les regroupent en trois catégories. Les catégories qu'ils dénomment «linguistique ${ }^{3}$ » et «didactique et pédagogie ${ }^{4}$ » représentent chacune $42 \%$ de l'ensemble, alors que la catégorie « culture et civilisation ${ }^{5}$ » représente seulement $16 \%$ des publications. Les auteurs concluent leur 
article par une invitation à poursuivre les recherches sur le sujet : «Cette réflexion sur les articles publiés dans ASp est partielle et donc partiale. Elle sera poursuivie ultérieurement » $(2008: 28)$.

3 En 2010, Jean-Louis Trouillon propose une analyse plus détaillée des domaines de spécialité abordés par les chercheurs et fait notamment ressortir que, aussi bien dans ASp que dans English for Specific Purposes, revue consacrée à l'anglais de spécialité dans une perspective de linguistique appliquée publiée par Elsevier, c'est l'approche didactique et pédagogique qui est la plus largement représentée. Il rappelle que «la plupart des travaux de recherche, et donc les publications qui en sont issues, sont le fait d'enseignants d'anglais de spécialité qui font part de leur expérience ou de leurs découvertes dans la matière qu'ils enseignent » $(2010: 52)$.

Ces deux analyses soulignent la prédominance de ce que nous appellerons le domaine des sciences (voir infra). Il semble donc pertinent de poursuivre l'étude des articles qui appartiennent à ce domaine, de façon à proposer un panorama aussi complet que possible des travaux menés jusqu'à présent par les chercheurs qui publient dans ASp et à comprendre quels facteurs ont pu influencer l'évolution de leurs travaux.

\section{Méthodologie}

\subsection{Hypothèse}

5 L'hypothèse émise pour cette étude est qu'il existe des liens entre le contexte de production des articles de recherche, les domaines de spécialité abordés par les chercheurs (spécifiquement ici celui des sciences) et l'approche qu'ils retiennent. Par contexte de production, nous entendons trois axes majeurs: l'actualité sur le plan politique, économique et scientifique notamment; la politique universitaire dans les établissements d'enseignement supérieur français et les missions de l'enseignant ou de l'enseignant-chercheur au sein de l'institution; enfin, l'avancement de la recherche en anglais de spécialité.

6 Cette même hypothèse est formulée par Guadalupe Aguado de Cea et Alejandro Curado Fuentes qui parlent de travaux marquants (pivotal works) qui ont accompagné le développement de l'anglais de spécialité en Espagne (Aguado de Cea \& Curado Fuentes 2012). Ils identifient également trois facteurs d'influence qui recoupent, dans une certaine mesure, ceux envisagés pour la présente étude : «administrative situation and change at the University", " teaching methods " et " research lines " (ibidem).

7 Pour mener à bien cette nouvelle étude, nous avons analysé les 120 articles $^{6}$ appartenant au domaine scientifique publiés dans la revue ASp de 1993 à 2012, soit un ensemble de soixante-deux numéros?

\subsection{Précisions terminologiques}

8 Pour rendre compte de l'ensemble des paramètres que nous avons pris en considération, il nous a paru nécessaire d'adopter une terminologie qui reprend, chaque fois que cela est possible, celle qui est généralement utilisée dans les études en anglais de spécialité, en particulier en France dans le cadre du GERAS. 


\subsubsection{Le domaine scientifique et les domaines de spécialité ou disciplines}

9 L'expression "domaine scientifique " est utilisée pour distinguer le domaine des sciences (que nous privilégions dans notre étude) de celui de l'économie, du droit ou de la médecine, par exemple.

10 Les termes "domaine de spécialité " ou "discipline » renvoient à la branche du domaine scientifique à laquelle s'intéressent les chercheurs en anglais de spécialité : l'informatique, la géologie, la biologie ou la physique par exemple (Mémet \& Van der Yeught 2008).

11 La dénomination "article du domaine scientifique» peut s'appliquer, selon les chercheurs en anglais de spécialité, à des publications portant sur des domaines spécialisés très variés. Pour cette étude, nous avons choisi d'analyser les articles relevant, selon nous, du domaine scientifique couvert par le groupe de travail "Sciences and Academia » du GERAS qui rassemble les enseignants et enseignantschercheurs souhaitant échanger sur «les discours scientifiques et universitaires en contexte anglophone ", conformément aux objectifs spécifiés sur la fiche d'identité du Groupe $^{8}$. À l'intérieur de cet ensemble, nous avons identifié douze domaines de spécialité, ou disciplines, qui sont : l'anglais pour l'ingénieur, l'astronomie, la biologie, la chimie, l'environnement, le génie électrique, le génie mécanique, la géologie, l'informatique, les mathématiques, la nutrition et la physique9 .

\subsubsection{L'approche}

Le terme " approche » est utilisé pour spécifier l'angle sous lequel le chercheur mène son analyse $\mathrm{e}^{10}$. Il renvoie à une catégorisation à la fois plus détaillée et plus large que celle qui est comprise par le terme «axe » dans son acception historique au GERAS (Perrin 1993 : viii) ${ }^{11}$.

Dans leur étude, M. Mémet et M. Van der Yeught (2008) regroupent les approches didactiques et technologiques que distingue le GERAS sous la catégorie « didactique et pédagogie ", celle-ci permettant d'inclure les articles qui présentent une expérience d'enseignement $^{12}$, sans toutefois l'étayer d'une réflexion didactique ${ }^{13}$. Ils proposent également la dénomination "culture et civilisation " qui a l'avantage, selon eux, de poser clairement le lien entre les études anglaises telles qu'elles sont définies actuellement et l'anglais de spécialité.

14 La catégorisation que propose J.-L. Trouillon (2010: 54) met en avant certaines approches comprises dans les quatre axes de recherche retenus par le GERAS linguistique, culturel, didactique et technologique - (Perrin 1993: viii), de façon à rendre plus précis les parallèles que l'on peut établir entre les publications des revues ASp et English for Specific Purposes. Il propose, en outre, la catégorie « institutionnel » qui vient au troisième rang des sujets d'étude pour les chercheurs qui publient dans English for Specific Purposes. Cette catégorie regroupe les travaux qui ont pour objet la mise en place administrative des enseignements d'anglais de spécialité, leur organisation et leur rôle au sein des établissements, ainsi que les contraintes institutionnelles comme l'évaluation par exemple.

15 Pour caractériser l'approche que les chercheurs adoptent dans les différents articles que nous avons étudiés, nous avons choisi d'opérer une synthèse entre la classification autour de laquelle s'organise le GERAS (linguistique, culturel, didactique et 
technologique) et une approche diachronique dans laquelle l'analyse peut être à la fois culturelle, discursive et linguistique (Charpy 2011). Cette classification montre combien les perspectives sont diverses et offrent la possibilité d'analyser un même objet sous différents angles.

\subsection{Les paramètres pris en compte}

\section{allemand ou en néerlandais. Il s'agissait surtout de vérifier si les écoles de pensée} nationales avaient pu influencer l'auteur de l'article ${ }^{15}$.

\section{Résultats et analyses}

\subsection{Nombre d'articles appartenant au domaine scientifique par année de publication de la revue $A S p$}

Le tableau 1 présente le nombre d'articles du domaine scientifique relevés dans la revue ASp et le nombre total d'articles publiés dans l'année (120 articles sur 519, soit $23,06 \%$ des articles publiés au cours des vingt années). 
Tableau 1. Nombre d'articles du domaine scientifiquepar rapport au nombre total d'articles de la revue

\begin{tabular}{|l|c|c|c|c|c|c|c|c|c|c|c|c|c|c|c|c|c|c|c|c|c|}
\hline $\begin{array}{c}\text { Nombre } \\
\text { articles }\end{array}$ & 1993 & 1994 & 1995 & 1996 & 1997 & 1998 & 1999 & 2000 & 2001 & 2002 & 2003 & 2004 & 2005 & 2006 & 2007 & 2008 & 2009 & 2010 & 2011 & 2012 & Total \\
\hline $\begin{array}{l}\text { du } \\
\text { domaine } \\
\text { scientifique }\end{array}$ & 24 & 14 & 6 & 11 & 17 & 7 & 7 & 6 & 3 & 5 & 3 & 4 & 0 & 3 & 1 & 2 & 3 & 3 & 0 & 0 & 119 \\
\hline $\begin{array}{l}\text { Nombre } \\
\text { total }\end{array}$ & 64 & 39 & 39 & 40 & 45 & 35 & 42 & 41 & 20 & 30 & 25 & 19 & 8 & 10 & 9 & 11 & 11 & 12 & 12 & 4 & 516 \\
\hline
\end{tabular}

L'introduction progressive d'un comité de lecture ${ }^{16}$ a induit une baisse sensible du nombre total d'articles publiés (de soixante-quatre en 1993 à une moyenne de dix à partir de 2005).

Le graphique présenté dans la figure 1 permet de visualiser la forte diminution du nombre d'articles du domaine scientifique publiés au fil des ans.

Figure 1. Pourcentage annuel d'articles du domaine scientifique par rapport au nombre total d'articles de la revue

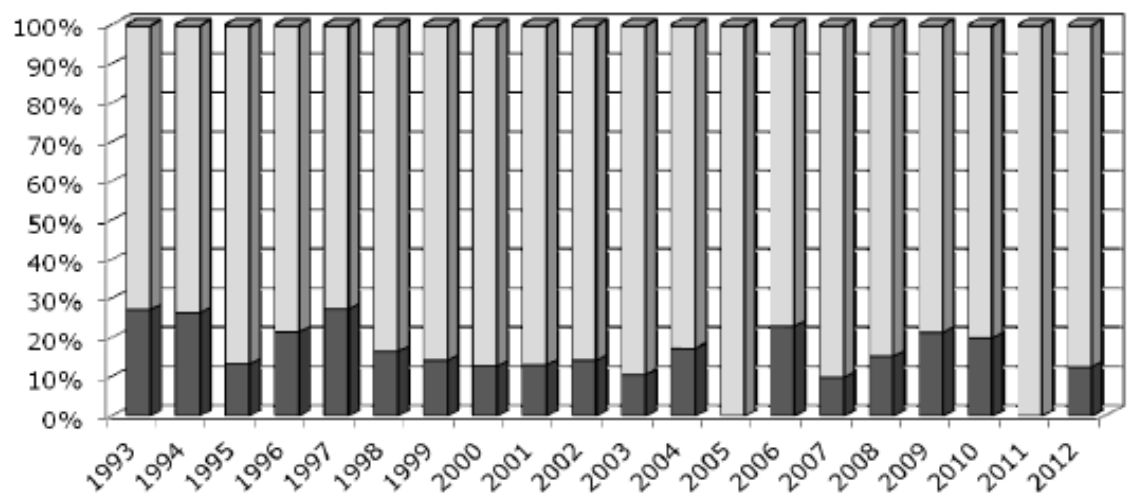

m $\%$ articles du domaine scientifique $\quad \neg \%$ autres articles

La diminution de la proportion d'articles appartenant au domaine scientifique est sensible à partir de 1998 ; les années 2005 et 2011 se distinguent même par l'absence de publication dans ce domaine. Nous pouvons envisager au moins trois hypothèses pour tenter d'expliquer leur importance relative dans ces premières années.

La première pourrait être que, lorsque l'anglais a été introduit dans les cursus, la plupart des postes d'enseignant d'anglais ont été créés dans les établissements scientifiques ${ }^{17}$. Le tableau 2 indique le pourcentage du nombre d'auteurs exerçant dans un établissement scientifique, par rapport au nombre total d'articles de la revue. Il montre la proportion relativement élevée d'auteurs enseignant dans ce domaine.

Tableau 2. Proportion des auteurs exerçant dans un établissement scientifique par rapport au nombre total d'articles de la revue

\begin{tabular}{|c|c|}
\hline Années & $\%$ \\
\hline 1993 & $31,25 \%$ \\
\hline 1994 & $28,21 \%$ \\
\hline 1995 & $10,26 \%$ \\
\hline 1996 & $20,00 \%$ \\
\hline 1997 & $31,91 \%$ \\
\hline 1998 & $17,14 \%$ \\
\hline 1999 & $16,67 \%$ \\
\hline 2000 & $12,20 \%$ \\
\hline
\end{tabular}



majoritairement des enseignants ou enseignants-chercheurs en exercice dans des établissements scientifiques ou à composante scientifique ainsi que le montre le tableau 3.

Tableau 3. Proportion des auteurs d'articles du domaine scientifique exerçant dans un établissement scientifique

\begin{tabular}{|c|c|}
\hline Années & $\%$ \\
\hline 1993 & $83,33 \%$ \\
\hline 1994 & $78,57 \%$ \\
\hline 1995 & $66,67 \%$ \\
\hline 1996 & $72,73 \%$ \\
\hline 1997 & $88,24 \%$ \\
\hline 1998 & $100,00 \%$ \\
\hline 1999 & $100,00 \%$ \\
\hline 2000 & $83,33 \%$ \\
\hline
\end{tabular}

Pour comprendre l'évolution du nombre de publications portant sur le domaine scientifique au fil des ans et, en particulier, la différence significative du nombre d'articles publiés d'une année à l'autre, nous avons aussi cherché à déterminer dans quelle mesure les orientations posées par le colloque du GERAS et la thématique de la revue ASp publiée la même année ont pu orienter les travaux des chercheurs. L'année 1995 est notable à cet égard: on y relève six publications seulement, alors que les années précédentes et suivantes, ce nombre était largement supérieur (voir tableau 1). Le thème du colloque du GERAS, "Langue de spécialité - Convergences, divergences ", n'avait apparemment guère suscité d'interventions de chercheurs en anglais scientifique. En revanche, en 2000, le colloque dont le thème était « Textes/Images dans les discours spécialisés " a retenu l'intérêt des chercheurs en anglais scientifique puisque le numéro 27-30 de la revue ASp comprend une section intitulée « Approches du discours scientifique ", les autres parties de l'ouvrage ne faisant référence à aucun discours de spécialité particulier. 


\begin{abstract}
spécialité scientifique diminue, leur pourcentage par rapport à l'ensemble des articles reste toujours inscrit à l'intérieur d'une fourchette comprise entre $12 \%$ et $27 \%$, soit environ $20 \%$ en moyenne depuis 2005.
\end{abstract}

D'une façon générale, bien que le nombre de publications portant sur des domaines de

\title{
2.2. Thèmes majeurs des articles du corpus
}

La classification des travaux des chercheurs en fonction du thème majeur qu'ils abordent renvoie à la distinction en deux secteurs qui recouvrent les deux orientations du GERAS : l'anglais de spécialité (les travaux qui portent sur la recherche théorique), et l'anglais pour spécialistes d'autres disciplines (les publications liées à l'enseignement). Par ailleurs, une faible proportion d'articles propose une réflexion sur le positionnement du chercheur par rapport à l'institution. La figure 2 montre la répartition des travaux des chercheurs selon ces trois secteurs: enseignement, recherche et institutionnel.

Figure 2. Secteurs représentés dans $A S p$

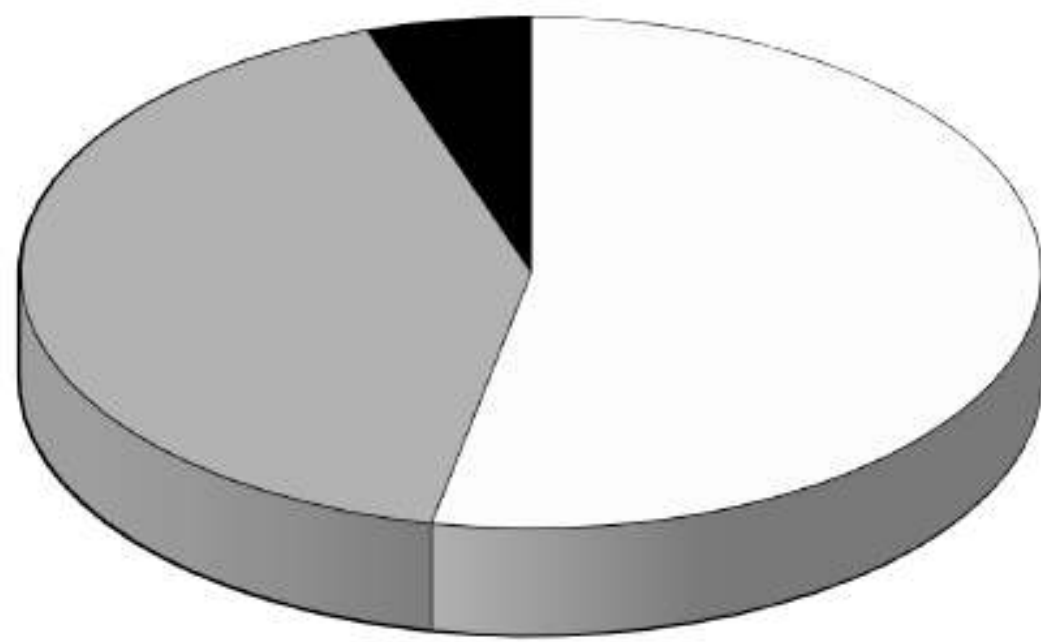

$\square$ Enseignement $\quad$ Recherche $\quad$ Institutionnel

Dans de nombreux articles, principalement dans les premières années de la revue, la recherche théorique amène parfois à une application pratique en cours. Nous avons alors classé l'article dans un seul secteur, en privilégiant celui dans lequel les apports nous paraissaient majeurs.

En revanche, afin d'affiner les résultats, nous avons, pour la suite de notre analyse, tenu compte de la diversité des approches à l'intérieur d'un même article ce qui explique certains écarts, en particulier dans les résultats présentés dans les tableaux 5, 6 et 7.

La figure 3, qui présente l'évolution de la langue de rédaction des articles au fil des ans, constitue une première étape dans l'analyse de l'impact de la recherche anglo-saxonne et française sur les auteurs. 
Figure 3. Langue de rédaction de l'article

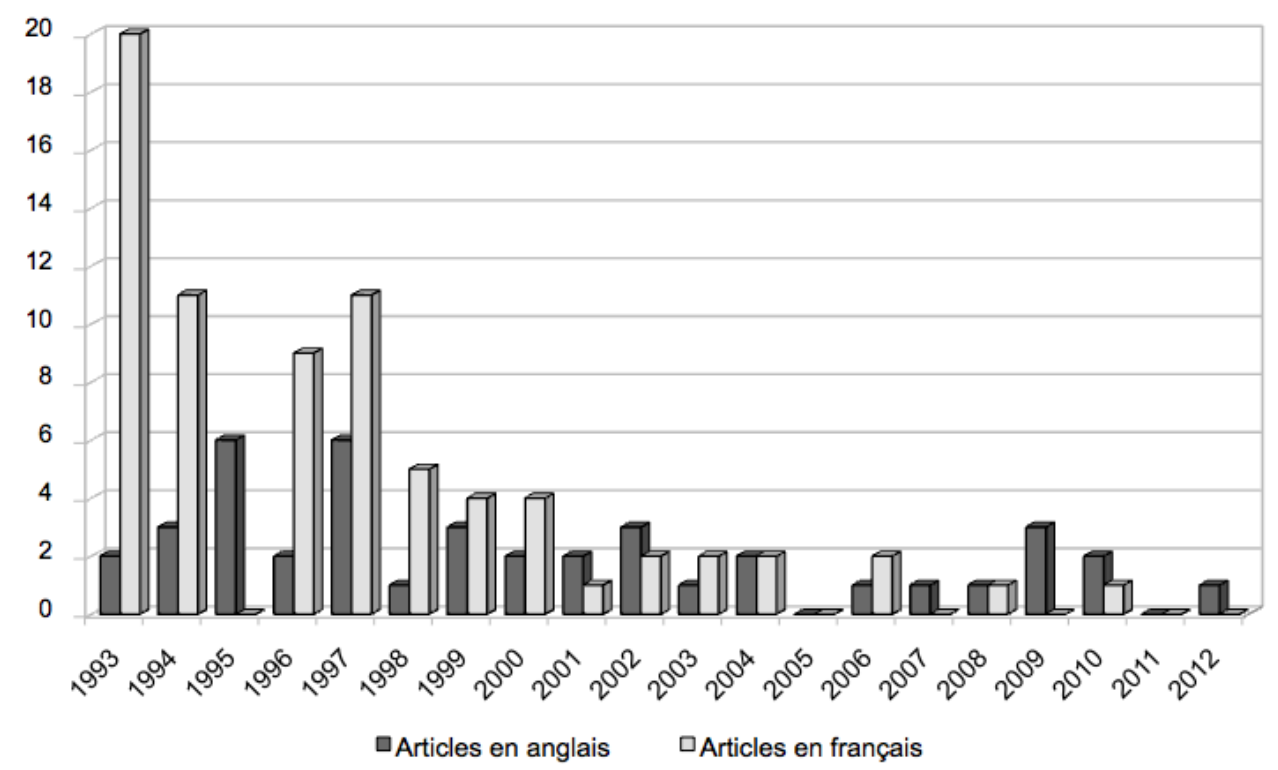

Entre 1993 et 2000, à l'exception de 1995 qui ne présente aucun article en français ${ }^{18}$, les auteurs ont très majoritairement rédigé en langue française (66 articles sur 92 soit $71,73 \%$ ). Par la suite, la tendance tend à s'inverser (17 articles sur 28 soit $57,14 \%$ sont rédigés en anglais).

Cette tendance, observée à partir du tournant du siècle (et qu'il faudrait vérifier dans l'ensemble des articles et non uniquement dans les articles du domaine scientifique), est probablement due à un désir d'ouverture à l'international de la part des auteurs. Comme dans toutes les autres disciplines universitaires les articles rédigés en français sont, en effet, peu cités dans le monde non francophone de la recherche. De nos jours, ils sont également relativement moins reconnus en France par les différentes instances d'évaluation de la recherche; la $11^{\mathrm{e}}$ section du CNU recommande par ailleurs la présentation de publications en anglais.

\subsection{Analyse des bibliographies}

L'analyse de l'origine des références citées par les auteurs montre que, sur un total de 1855 références bibliographiques, 1316 (soit 71,6\%) renvoient à des auteurs anglophones et 536 (soit $28,4 \%$ ) à des auteurs francophones, ce qui témoigne de la prépondérance des sources anglophones dans les travaux des chercheurs.

41 Pour quantifier cette influence en fonction de la langue de rédaction des articles (anglais ou français), nous avons établi deux graphiques, l'un à partir des articles rédigés en anglais (figure 4), l'autre à partir des articles rédigés en français (figure 5). 
Figure 4. Langue des références citées en bibliographie dans les articles rédigés en anglais

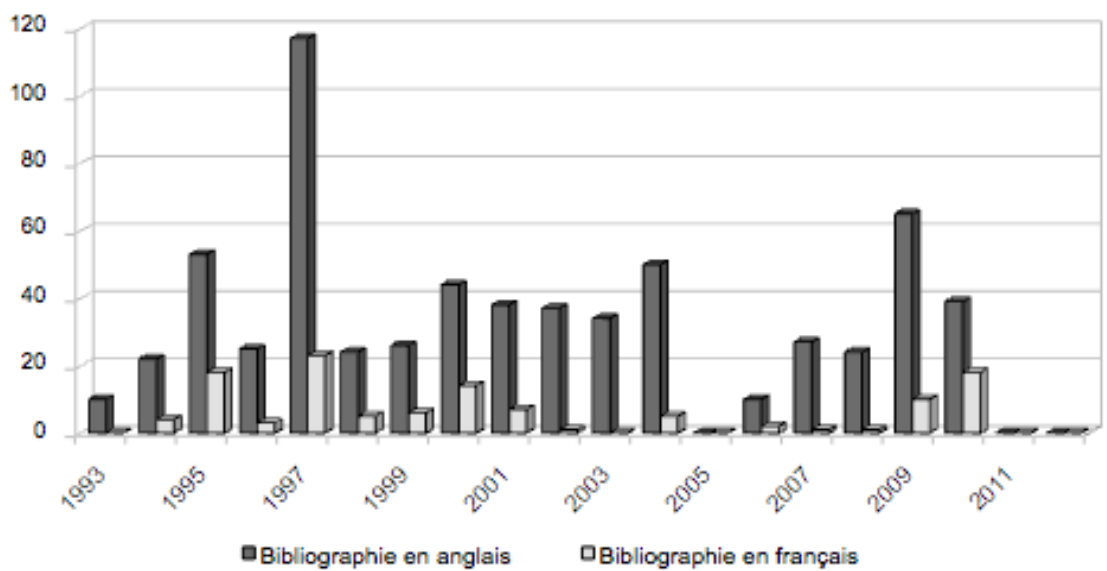

Ce graphique permet, sans surprise, de visualiser la prééminence des sources anglophones dans les articles rédigés en anglais: elles représentent 84,53\% des citations alors que les sources francophones ne représentent que $15,4 \%$ des références bibliographiques. Ces dernières comprennent des thèses ou des mémoires rédigés en France, les auteurs étant généralement des anglophones ayant dû se conformer aux normes universitaires françaises.

La figure 5, qui présente les résultats de l'analyse des bibliographies dans les articles francophones, montre que les sources anglophones restent majoritaires. Elles représentent encore $62,18 \%$ du total.

Figure 5. Langue des références citées en bibliographie dans les articles rédigés en français

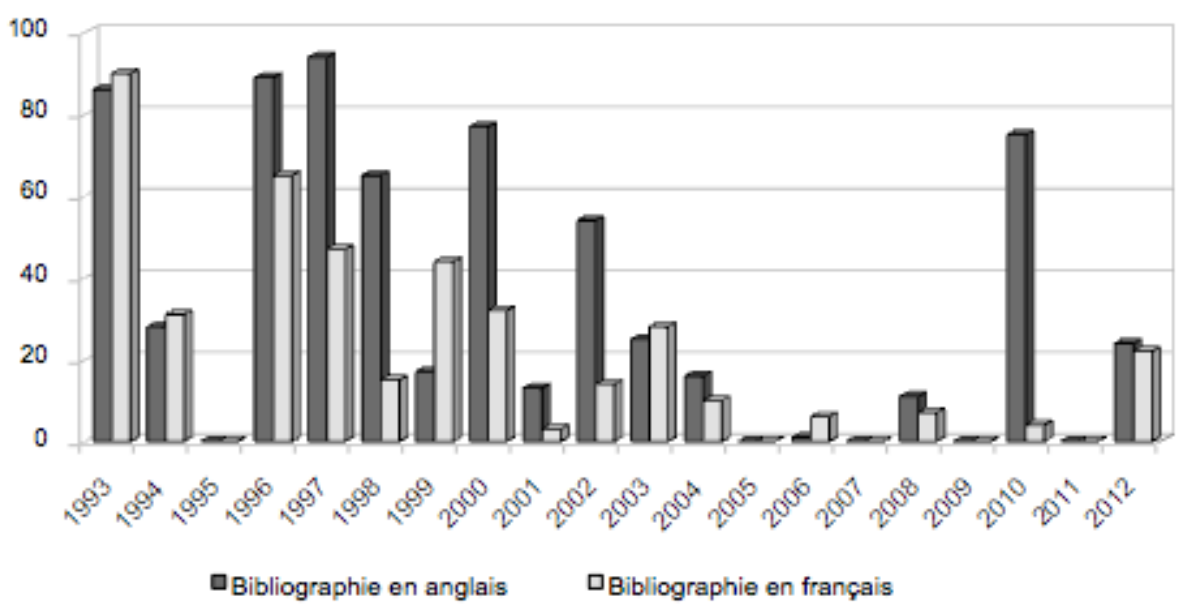

Afin de confirmer ou d'infirmer l'hypothèse selon laquelle la prédominance des sources anglophones s'expliquerait par les nombreuses références aux grands auteurs anglosaxons généralistes de l'ESP, nous avons procédé à un relevé nominatif de ces sources, au nombre de 419, ainsi que des dates de citation. Le tableau 4 présente les données recueillies pour les douze auteurs les plus fréquemment cités, c'est-à-dire ceux qui apparaissent dans au moins cinq articles différents; plusieurs ouvrages d'un même auteur peuvent en effet être cités dans la bibliographie d'un même article. 
Tableau 4. Principales sources bibliographiques citées par année

\begin{tabular}{|c|c|c|c|c|c|c|c|c|c|c|c|c|c|c|c|c|c|c|c|c|c|c|}
\hline \multirow{2}{*}{ Auteurs } & \multirow{2}{*}{$\begin{array}{c}\begin{array}{c}\text { Nombre } \\
\text { d'articles }\end{array} \\
\end{array}$} & \multirow{2}{*}{$\begin{array}{c}\text { Nombre de } \\
\text { citations }\end{array}$} & \multicolumn{20}{|c|}{ Années de citation } \\
\hline & & & 293 & 394 & mos & nos & 299 & 29: & \begin{tabular}{|l|l|} 
m95 \\
\end{tabular} & 2000 & 2001 & 2002 & 2003 & 2004 & 2005 & 2006 & 2000 & 2000 & 2009 & 200 & 20112 & 200 \\
\hline J.Swales & 21 & 27 & $\bar{x}$ & & & $x$ & $\bar{x}$ & $x$ & $x$ & $\bar{x}$ & & $\bar{x}$ & & $\bar{x}$ & & $\mathrm{x}$ & & & & $x$ & & $\mathrm{x}$ \\
\hline G.Myers & II & In & & $\mathrm{x}$ & & $x$ & & \begin{tabular}{|l|l|}
$x$ \\
\end{tabular} & $\bar{x}$ & $\bar{x}$ & & $\mathrm{x}$ & & & & & & & & & & \\
\hline F.Salager-Meyer & 7 & $n$ & & $x$ & & $x$ & $x$ & $x$ & & & & $\mathrm{x}$ & & & & & & & & & & \\
\hline C.Bazerman & 5 & $\mathbf{n}$ & $x$ & $x$ & & $x$ & & $x$ & & $x$ & & $\mathrm{x}$ & & & & & & & & & & \\
\hline T.Dudley-Evans & 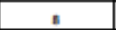 & $\mathbf{n}$ & $x$ & $x$ & & $x$ & & $x$ & $x$ & $\mathbf{x}$ & & & & & & & & & & & & \\
\hline B.Latour & 3 & 9 & & & & $x$ & & $\mathrm{x}$ & & $\mathbf{x}$ & & & & & & & & & & & & \\
\hline M. Halliday & 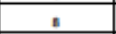 & $a$ & $\mathrm{x}$ & & & $x$ & & & $\mathrm{x}$ & $\mathrm{x}$ & & & & & & & & & & & & \\
\hline A. Johns & 3 & 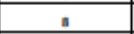 & & & & $x$ & & $x$ & & & & & & & & & & & & $x$ & & \\
\hline L. Trimble & 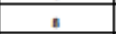 & a & $\mathrm{x}$ & & & $x$ & & $\mathbf{x}$ & & $x$ & & $\mathrm{x}$ & & & & & & & & & & \\
\hline K.Knorr-Certina & $s$ & 7 & & & & $\mathrm{x}$ & & $x$ & & $\mathrm{x}$ & & & & & & & & & & & & \\
\hline T.Miller & 3 & 7 & & $x$ & & $x$ & & $x$ & & $\mathrm{x}$ & & & & & & & & & & & & \\
\hline G.Leech & 3 & 8 & $x$ & & & & & & & \begin{tabular}{|l|l|}
$x$ \\
\end{tabular} & & $\mathrm{x}$ & & & & & & & & $\mathrm{x}$ & & \\
\hline
\end{tabular}

C'est effectivement l'un des auteurs de renom de l'ESP, J. Swales, qui est le plus fréquemment et le plus abondamment cité, soit vingt-sept fois dans vingt-et-un articles différents. Il est le seul à être mentionné régulièrement depuis la création de la revue, les autres chercheurs étant cités très fréquemment au cours des dix premières années puis plus du tout ${ }^{19}$.

Deux hypothèses au moins peuvent être avancées pour expliquer cette particularité : d'une part, certains chercheurs comme Tony Dudley-Evans, Thomas Miller ou Françoise Salager-Meyer par exemple, ont eux-mêmes publié des articles dans les premiers numéros de ASp et ce sont ces articles qui ont été cités dans les numéros suivants de la revue. D'autre part, un attrait passager pour certains sujets explique sans doute l'absence de références, par exemple, à Charles Bazerman ou à Greg Myers à partir du début du XXI ${ }^{e}$ siècle.

Quant à F. Salager-Meyer, elle est la seule non-anglophone de la liste à être citée (sept fois) en tant que source publiée en anglais ; ses articles figurent en effet régulièrement dans des revues anglophones, en particulier dans English for Specific Purposes ${ }^{20}$.

Cette étude fait ressortir le cas de Noam Chomsky qui n'apparait qu'une seule fois (Frost 2004) pour son ouvrage The Sound Pattern of English (1968), rédigé en collaboration avec Morris Halle. Si ce chercheur, qui a profondément marqué la linguistique moderne, n'est pas cité plus souvent, c'est peut-être parce que l'influence de la linguistique de l'énonciation sur les chercheurs francophones est telle qu'ils n'ont point senti le besoin de faire appel à une théorie assez éloignée de leurs préoccupations et parfois même considérée par certains comme dépassée ; Syntactic Structures date en effet de 1957, soit trente-six ans avant le premier numéro de ASp.

\subsection{Les domaines de spécialité scientifique les plus étudiés}

Pour comprendre en quoi le contexte de production, sur le plan de la politique universitaire notamment, a pu influer sur les axes de recherche suivis par les chercheurs, nous avons classé les articles par domaine de spécialité scientifique et par année (tableau 5). 
Tableau 5. Nombre d'articles par domaine et par année

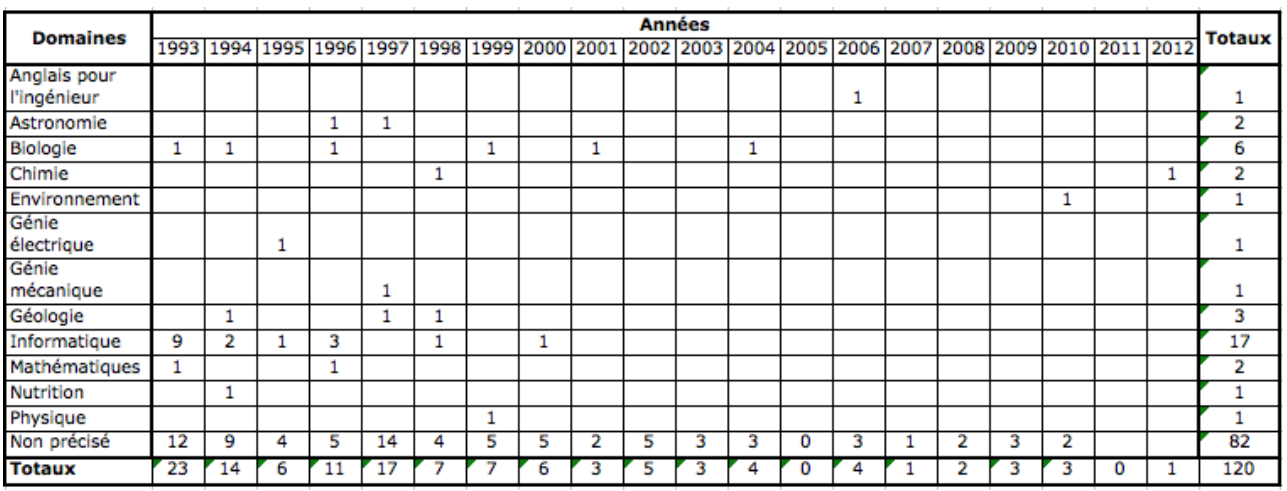

Quatre-vingt-deux articles sur 120 (soit 68,33\%) traitent des sciences en général, sans autre précision, cette tendance étant forte jusqu'en 2000 (58 articles entre 1993 et $2000 ; 24$ articles de 2001 à 2012).

51 Sans doute ce phénomène s'explique-t-il par l'influence des conditions dans lesquelles l'enseignement en anglais dans les filières scientifiques a été mis en place : il s'agissait, pour des enseignants dont la formation était généralement littéraire, d'aborder la question du discours scientifique et la différenciation entre les filières ne semblait pas être sensible dans les premiers temps. Par ailleurs, certains chercheurs, par exemple J. Martin $(1997$; 1998), s'étaient attachés au domaine conceptuel de la science et raisonnaient donc de façon globale.

La figure 6 illustre le nombre d'articles par domaine de spécialité et par discipline publiés de 1993 à décembre 2012 ; La catégorie « non précisé » (voir tableau 5), n'est pas prise en compte bien que largement majoritaire, de façon à offrir une lecture plus claire des données et à permettre de juger de l'importance relative des domaines traités. 
Figure 6. Nombre d'articles par domaine de spécialité et par discipline

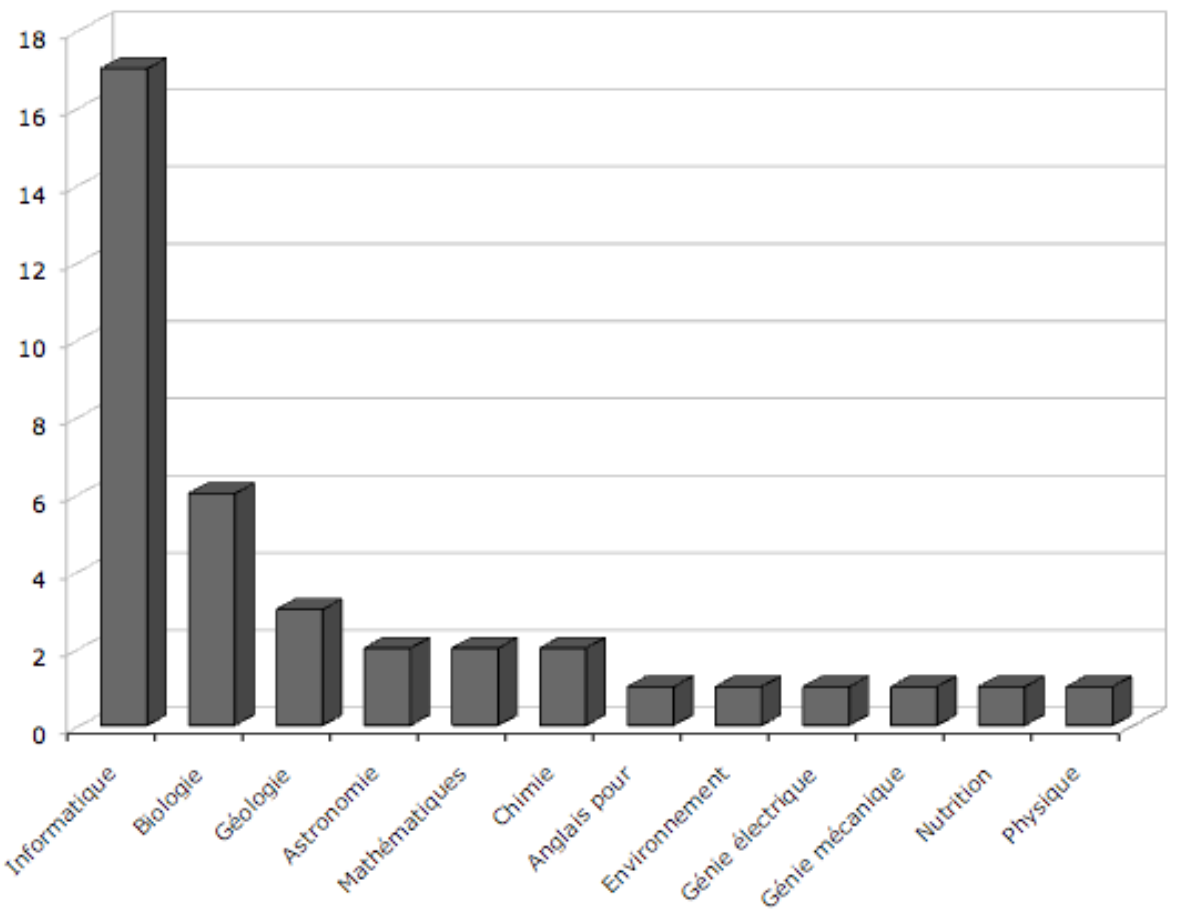

L'informatique est le domaine qui a le plus attiré les auteurs dans les premières années de la revue jusqu'en 2000 ( 17 articles sur 120 , soit 14,16\% des articles publiés). On note que, dès le premier numéro de la revue, l'informatique a été à l'origine de nombreux travaux (Pradeilles 1993; Boughedaoui 1993; Dechet 1993; Corbisier 1993; Mémet 1993) dans la mesure où les besoins en enseignement dans ce domaine étaient grands : de nombreux étudiants se dirigeaient alors dans cette voie nouvelle et professionnellement prometteuse. La documentation mise à leur disposition et à celle de leurs enseignants était à l'origine uniquement rédigée en anglais. Les IUT d'abord, puis les universités ont fait appel à de nombreux enseignants d'anglais qui ont dû aussi bien créer des enseignements adaptés que s'interroger sur les spécificités de l'anglais de l'informatique. Toutes ces réflexions, pratiques mais aussi théoriques, sont à l'origine des premières publications ${ }^{21}$.

Parmi les autres disciplines, seule la biologie (six articles soit $5 \%$ ) se détache légèrement. Certains chercheurs ont été en effet particulièrement productifs à l'intérieur d'une même spécialité pour répondre aux contraintes propres de leur institution pendant un temps donné22 alors que d'autres se sont spécialisés dans un certain type de sujet ou d'approche ${ }^{23}$.

Certaines disciplines comme la physique ou la chimie, pourtant largement représentées dans les établissements d'enseignement supérieur scientifique, n'ont suscité que peu d'intérêt jusqu'à ce jour.

\subsection{Les approches retenues par les auteurs}

Pour vérifier l'hypothèse selon laquelle les approches retenues par les auteurs ont évolué au fil du temps, au fur et à mesure qu'évoluait la recherche théorique en anglais 
de spécialité elle-même, nous avons mis en parallèle les disciplines ou les domaines de spécialité traités et le nombre d'articles qui leur sont consacrés puis nous avons croisé ces données avec les approches retenues par les auteurs des articles (tableau 6).

Tableau 6. Approches par domaine et par discipline

\begin{tabular}{|c|c|c|c|c|c|c|c|c|c|}
\hline \multirow{2}{*}{ Domaines } & \multicolumn{8}{|c|}{ Approches } & \multirow{2}{*}{ Totaux } \\
\hline & Culture & Diachronie & Enseignement & Analyse du discours & Grammaire & Phonologie & Terminologie & TICE & \\
\hline Anglais pour /ingénieur & 1 & & & & & & & & 1 \\
\hline Astronomie & & & & 1 & 1 & & & & 2 \\
\hline Biologie & 1 & 1 & 1 & 1 & 2 & & 2 & & 8 \\
\hline Chimie & & & & & & & 1 & & 1 \\
\hline Environnement & & & & 1 & & & & & 1 \\
\hline Génie électrique & & & 1 & & & & & & 1 \\
\hline Génie mécanique & & & & 1 & & & & & 1 \\
\hline Géologie & 1 & & & 2 & 1 & & & & 4 \\
\hline Informatique & & & 11 & 2 & 2 & & 2 & & 17 \\
\hline Mathématiques & & & & & 1 & & 1 & & 2 \\
\hline Nutrition & 1 & & & & 1 & & & & 2 \\
\hline Physique & & & 1 & & & & & & 1 \\
\hline Non précisé & 10 & 7 & 36 & 31 & 10 & 1 & 3 & 2 & 100 \\
\hline Totaux & 14 & 8 & 50 & 39 & 18 & 1 & 9 & 2 & \\
\hline
\end{tabular}

Ce tableau permet notamment de visualiser combien, pour l'informatique, le lien entre le contexte de production tel que nous l'avons décrit plus haut et l'approche privilégiée par les chercheurs est fort puisque c'est la discipline qui a suscité le plus grand nombre d'articles selon une approche didactique.

Rappelons toutefois qu'un même article peut s'articuler autour de deux, voire trois approches. John Humbley et Maria M. Negroni (2004), par exemple, abordent leur corpus en biologie selon une approche à la fois grammaticale et terminologique. Il n'est pas rare non plus qu'une analyse grammaticale ou phonologique débouche sur une application pratique en cours.

La figure 7 montre les proportions relatives entre ces différentes approches.

Figure 7. Nombre total d'articles par approche

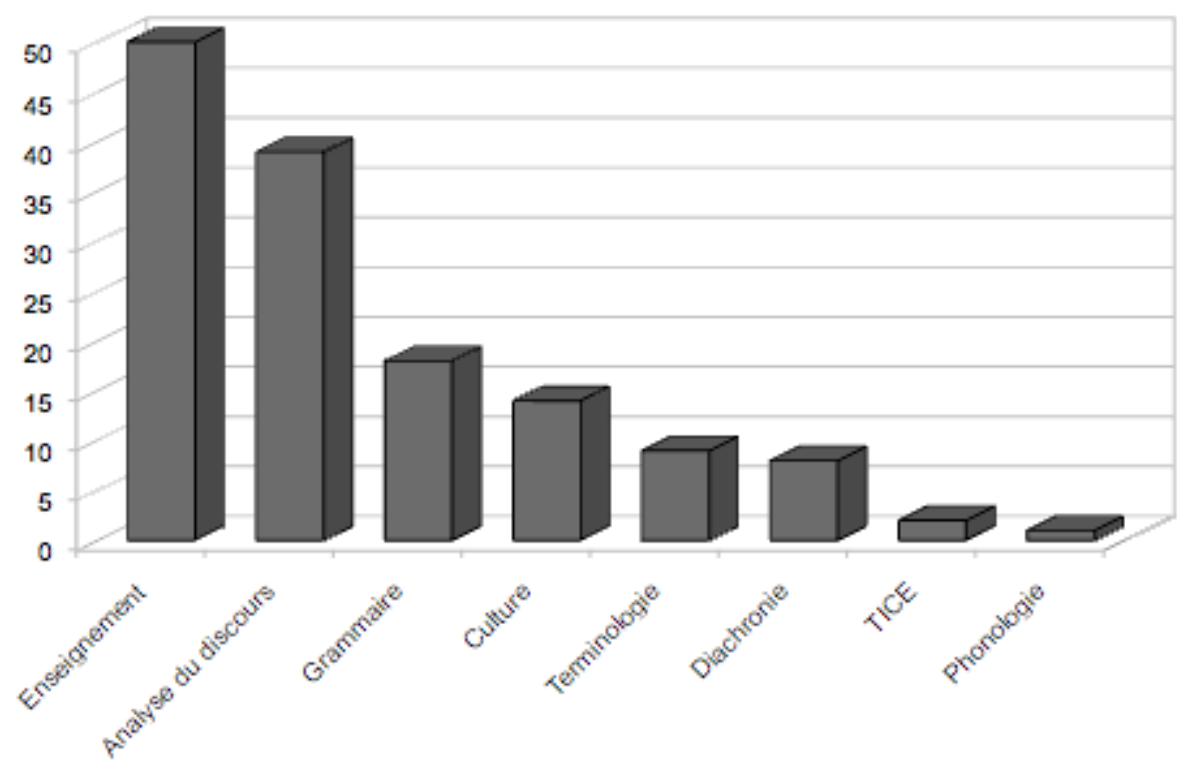

Les premiers travaux de recherche en anglais de spécialité étaient surtout le fait d'auteurs confrontés à l'enseignement d'une nouvelle discipline. Le graphique fait 
apparaître, sans surprise, une forte proportion d'articles s'inscrivant dans cette approche (cinquante sur 140 soit $35,7 \%$ ). Le tableau 7 permet de visualiser la répartition de ces différentes approches dans le temps.

Tableau 7. Nombre d'articles par approche et par année

\begin{tabular}{|c|c|c|c|c|c|c|c|c|c|c|c|c|c|c|c|c|c|c|c|c|c|}
\hline Approches & & & & & & & & & & Ant & & & & & & & & & & & Totaux \\
\hline Fqpiocties & 1983 & 1994 & 1925 & 1996 & & 1828 & & & 2001 & & & 2004 & 2005 & 2008 & 2007 & 2006 & 2008 & 2010 & 2011 & 12012 & Tolow \\
\hline Enseignement & 18 & 7 & 3 & 5 & 7 & & 3 & 2 & & 2 & 1 & 1 & & & 1 & 2 & & 11 & & & 51 \\
\hline Analyse du discours & 4 & 2 & 1 & 2 & 8 & 5 & 4 & 4 & 1 & 1 & \begin{tabular}{|l|}
2 \\
\end{tabular} & & & 1 & & & 2 & 2 & & & 39 \\
\hline Grammaire & 4 & 3 & 1 & & 2 & 1 & & 1 & 1 & 2 & & 2 & & 1 & & & & & & & 18 \\
\hline Culture & 1 & 6 & & & & & & & & & & 2 & & 1 & & & 3 & 1 & & & 14 \\
\hline Terminologie & 2 & & 1 & 3 & & & & & 1 & & & 1 & & & & & & & & 1 & 9 \\
\hline Diachronie & & & & & 1 & 1 & & 1 & & & & 1 & & 1 & & & 2 & 1 & & & 8 \\
\hline TICE & & & & & & & & & & & 1 & & & 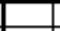 & & 1 & & 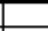 & & & 2 \\
\hline Phonologie & 1 & & & & & & & & & & & & & & & & & & & & 1 \\
\hline
\end{tabular}

61 En 1993, par exemple, quinze articles sur vingt-quatre, soit près des deux tiers, sont directement consacrés à l'enseignement, mais il peut y avoir plusieurs approches pour un même article. En 1994 et 1995, la proportion est encore de 50 \% (respectivement sept articles sur quatorze et trois articles sur six), En 1996, il en va pratiquement de même avec cinq articles sur onze. On observe une baisse progressive du nombre d'articles consacrés à l'enseignement à partir de 1997. Certaines années (1998, 2001, 2005, 2006, 2009 et 2012) cette approche est même totalement absente. Bien que la moyenne sur l'ensemble des vingt années de publication s'établisse à $42 \%$, la baisse observée à partir de 1998 (28,26 \% en moyenne entre 1998 et 2012) peut s'expliquer par la disparition des articles consacrés à des expériences pédagogiques, principalement sous l'influence des rédacteurs en chef de la revue qui ont orienté leur choix éditorial vers les articles de recherche et introduit le filtre du comité scientifique de lecture.

L'analyse du discours (trente-neuf articles sur 120 soit 32,5\%) représente une part importante des travaux de recherche dans le domaine scientifique, suivant ainsi l'une des grandes tendances de la recherche en anglais de spécialité.

Il faut noter la présence non négligeable d'une approche culturelle dès le premier numéro de la revue (Brouzeng 1993). L'intérêt pour cette approche s'exprime fortement, dès l'année suivante, au travers du thème du colloque du GERAS « Langue de spécialité et culture », le volume des $n^{\circ}$ 5-6 de la revue étant constitué des actes du colloque.

64 L'approche grammaticale est bien représentée. La majorité des articles porte sur la grammaire en général et, plus rarement, sur des points précis : les adjectifs composés (Boughedaoui 1997), les relatifs (Léonarduzzi 1998 ; Williams et al. 2002), les temps et aspects (Labrosse 2000 ; Banks 2006).

65 L'approche diachronique est privilégiée par quelques auteurs dont D. Banks $(1997,2004$, 2006, 2009, 2010) ${ }^{24}$, Anne Magnet (1999 et 2000), F. Salager-Meyer (1998) et Jean-Pierre Charpy (2011).

66 On pourra en revanche s'étonner de la faible présence des TICE (Laffont et al. 2003; Frost 2008) qui ont fait par ailleurs l'objet de nombreuses publications ${ }^{25}$ sans prendre toutefois les sciences comme domaine d'application. 


\subsubsection{Les compétences et sujets étudiés par les chercheurs en Lansad} En conséquence, il semble pertinent de chercher à identifier sur quelles compétences ou sujets les articles soumis à la revue ASp ont porté en priorité (figure 8).

Figure 8. Compétences et sujets traités dans le secteur Lansad

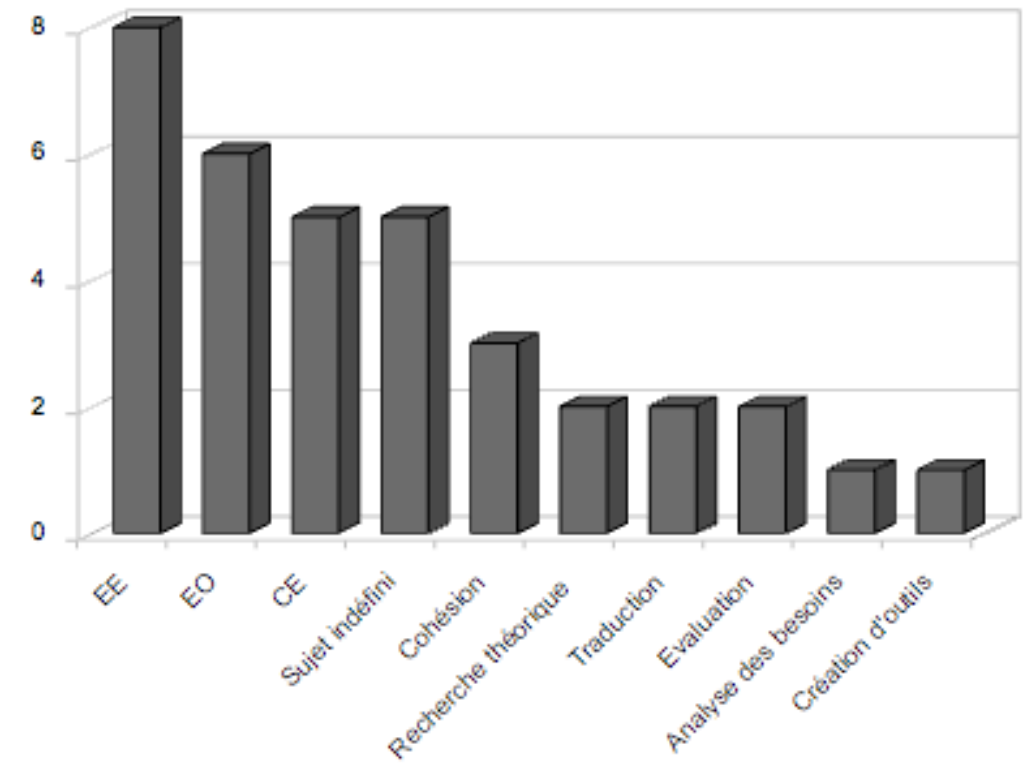

Ce graphique illustre la prédominance de travaux sur l'expression écrite (EE), l'expression orale (EO) et la compréhension écrite (CE) ; il met en évidence l'absence de recherche menée sur la compréhension orale.

Plusieurs éléments peuvent expliquer le fait que l'expression écrite mobilise une très grande partie de l'attention des chercheurs: le lien entre anglicistes et enseignantschercheurs spécialistes d'autres disciplines qui sollicitent l'aide de leurs collègues linguistes; la lassitude des enseignants face aux nombreuses fautes trouvées dans des copies de devoirs ou d'examens et le désir d'apporter des solutions efficaces à ce problème ; la nécessité d'enseigner l'écriture de genres professionnels (comptes rendus d'expériences, rapports de stages, abstracts, articles de recherche) ; la simplicité relative de constitution et de traitement d'un corpus écrit, par opposition à un corpus de documents sonores par exemple.

La place de l'expression orale pourrait surprendre si l'on songe aux objectifs professionnels alors présentés comme prioritaires dans certaines filières et à certains niveaux d'études. Dans les universités, l'enseignement de l'anglais était, à ses débuts, généralement restreint au DEUG, voire uniquement à la première année de DEUG. À ce stade de leurs études, les étudiants devaient prioritairement être capables de lire la documentation proposée par leurs enseignants des disciplines scientifiques et il n'était aucunement question de les préparer à assister à des colloques ou à partir en mission dans un laboratoire étranger. Il en allait de même dans les IUT et dans les classes de techniciens supérieurs préparant au BTS. Dans ces établissements toutefois, la taille plus réduite des groupes de travaux dirigés et les heures de cours plus nombreuses permettaient éventuellement la pratique orale. Dans les écoles d'ingénieurs, les 
objectifs étaient différents, l'oral faisant partie des priorités pour l'insertion dans la vie professionnelle.

71 La compréhension écrite est abordée dans cinq articles seulement, mais les thèmes que ces travaux développent sont parfaitement ciblés puisqu'ils portent sur l'acquisition de stratégies de lecture. Dans le cadre d'un apprentissage de la langue à visée professionnelle, les enseignants-chercheurs répondaient ainsi à la nécessité de former les étudiants à la consultation de bibliographies ou de modes d'emplois de matériels.

Il faut sans doute voir dans la place prépondérante occupée par l'expression, qu'elle soit écrite ou orale, l'influence de la formation professionnelle initiale des enseignants d'anglais, souvent issus du second degré. Les instructions ministérielles à destination des collèges et lycées accordent une très grande place à l'expression, en particulier à l'expression orale. Ce besoin n'est pas nécessairement celui de tous les étudiants du secteur Lansad.

\subsubsection{Les types d'approche privilégiés dans le cadre de l'analyse du discours}

Les travaux portant sur l'analyse du discours sont également nombreux, comme nous l'avons montré précédemment (voir tableau 7). La figure 9, qui présente les types d'analyses privilégiées dans ce cadre, permet de vérifier l'hypothèse selon laquelle c'est l'étude du genre qui a largement mobilisé les chercheurs. Ce point corrobore la remarque faite à la section 2.4 concernant $\mathrm{J}$. Swales : il reste l'auteur auquel il est fait référence le plus souvent et ses travaux ont fortement marqué les chercheurs s'intéressant à la problématique du genre dans le discours scientifique.

Figure 9. Détail de l'analyse du discours

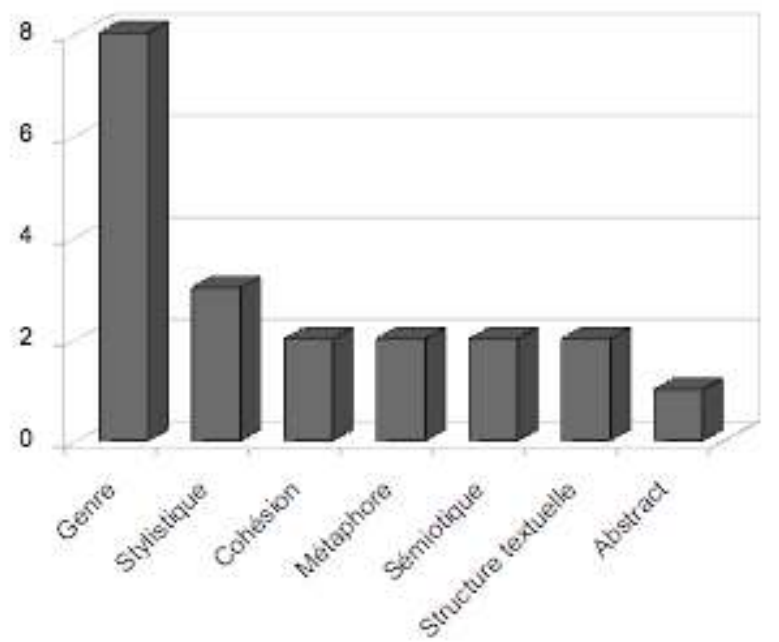

Le genre et la stylistique se détachent de l'ensemble des autres approches. Ces deux orientations, qui rejoignent les préoccupations d'une partie de la communauté des spécialistes de l'anglistique, renforcent les liens que l'on peut établir au niveau de la recherche entre anglais général et anglais de spécialité.

Le nombre des publications qui analysent les écrits scientifiques de façon à en dégager la spécificité et à tracer les contours d'une langue de spécialité augmente progressivement. Si elles ne sont que trois en 1993 et $1994^{26}$, on en compte dix en 1997 et sept en $1998^{27}$. Les chercheurs en anglais de spécialité, selon des centres d'intérêt qui 
leur sont propres, choisissent une approche ou une autre. Pourtant des tendances fortes se dessinent, révélatrices des courants d'influence. On note par exemple que, en 1997, sur les dix articles mentionnés précédemment, huit sont traités sous l'angle de l'analyse du discours. À cette période, nombreux sont les chercheurs anglicistes français qui sont influencés par les principaux travaux de Louis Trimble (1985) et de J. Swales (1990) notamment, ainsi que par la théorie énonciative des linguistes français, ou encore par les travaux de chercheurs en sciences du langage (Laffont 2005). Cette influence perdure puisque l'approche discursive est toujours largement représentée.

\section{Conclusion}

76 Les publications dans la revue ASp se répartissent très tôt dans deux secteurs majeurs, l'anglais de spécialité et l'anglais pour spécialistes d'autres disciplines, même si une distinction claire entre ces secteurs n'est formalisée qu'en $2007^{28}$.

77 Les enseignants et enseignants-chercheurs confrontés à la mise en place d'enseignements d'anglais de spécialité ont été amenés à réfléchir à de nouvelles approches et leurs travaux ont porté majoritairement sur les pratiques d'enseignement. Notre étude montre que, pour le domaine des sciences, cette dynamique semble avoir considérablement ralenti à partir de 1997.

78 Sans doute la cohabitation d'enseignants et enseignants-chercheurs spécialistes d'anglais avec des spécialistes d'autres disciplines a-t-elle permis de favoriser une collaboration en lien avec le besoin croissant de ces derniers de publier en anglais. Les premiers travaux de recherche qui analysent les publications scientifiques, spécifiquement dans le but d'aider les spécialistes d'autres disciplines à écrire des articles dans une langue qui corresponde aux exigences des revues scientifiques internationales, ont été publiés en 1994 (Crosnier; Birch) et ils sont l'apanage d'un groupe relativement réduit de chercheurs.

79 Nombreux sont les chercheurs qui abordent le domaine scientifique sous l'angle de l'analyse du discours. L'intérêt pour cette approche apparait dès les premières publications dans la revue ASp et reste soutenu puisque l'on compte encore quatre articles entre 2009 et 2010.

80 Nous avons aussi noté la présence d'une approche culturelle de l'anglais de spécialité dès la première année de la revue. Elle reste d'actualité mais elle est peu fréquente, et elle est principalement articulée avec des analyses de type diachronique, comme dans les travaux de D. Banks (2012) et de J.-P. Charpy (2011).

81 La FASP (Fiction à substrat professionnel), qui a par ailleurs été largement étudiée, en particulier dans les domaines du droit, de l'économie et de la médecine, a suscité peu de publications de chercheurs en anglais scientifique dans ASp. Seule Stéphanie Genty en propose une approche en 2009, alors que le concept est défini par Michel Petit en $1999^{29}$. Il est vrai que les « technothrillers » portent davantage sur les conséquences des découvertes de la science sur la politique que sur les milieux scientifiques anglophones eux-mêmes. Ces ouvrages ne présentent donc pas, pour l'enseignement, le même intérêt que ceux qui abordent les autres domaines de spécialité.

82 Les résultats présentés dans cette étude permettent d'identifier dans quels domaines de spécialité les chercheurs qui publient dans la revue ASp ont travaillé jusqu'à ce jour et quelles approches ils ont privilégiées. Les domaines ou disciplines qui apparaissent 
comme vacants dans notre étude (tableau 6) semblent représenter autant de pistes que les chercheurs qui s'intéressent à l'anglais scientifique pourraient envisager d'explorer à l'avenir. Toutefois, il faudrait préalablement compléter ces résultats en effectuant une étude similaire des Cahiers de l'APLIUT en France et d'Ibérica en Espagne avant de tirer des conclusions définitives. On peut également imaginer que les classifications qui semblent pertinentes actuellement pour rendre compte du travail de recherche effectué jusqu'à ces dernières années dans le cadre de l'anglais de spécialité soient reconsidérées, étant donnée l'évolution de la recherche. Marie-Agnès Détourbe rappelle «la volonté de certains chercheurs de dépasser la seule perspective d'enseignement pour orienter leur recherche » (2012: 7) et souligne que les chercheurs privilégient désormais volontiers ce qu'elle appelle « une perspective globale» (2012: 7). Certains chercheurs semblent en effet se tourner désormais vers des objets complexes plus larges comme le monde de l'enseignement supérieur (Détourbe 2011) ou celui de l'environnement (Biros 2011) en contexte anglophone ${ }^{30}$. Il est probable que l'on assiste, dans les années à venir, à une évolution du périmètre même de l'anglais scientifique et, plus largement, de l'anglais de spécialité qui amène les chercheurs à adopter une démarche interdisciplinaire et à intégrer les apports des spécialistes d'autres disciplines.

\section{BIBLIOGRAPHIE}

\section{Références des sources primaires}

ASp 1 à 62, 1993-2012. <http://asp.revues.org/> site consulté le 22/12/2012.

Banks, David. 1997. « Your very first ESP text (wherein Chaucer explaineth the astrolabe) ». ASp 15-18, 451-460.

Banks, David. 2004. « Philosophy, science and the establishment of science as a profession ». ASp 43-44, 169-80.

Banks, David. 2006. « The simple present and the simple past in the scientific journal article: a diachronic perspective ». ASp 49-50, 119-135.

Banks, David. 2009. « Starting science in the vernacular. Notes on some early issues of the Philosophical Transactions and the Journal des Sçavants, 1665-1700 ». ASp 55, 5-22.

Banks, David. 2009. "Creating a specialized discourse: the case of the Philosophical Transactions ». ASp 56, 29-44.

Banks, David. 2010. « Transitivity and thematic structure in some early issues of the Philosophical Transactions ». ASp 58, 57-71.

Banks, David. 2012. « Diachronic ESP: at the interface of linguistics and cultural studies ». ASp 61, $55-70$.

Birch, Sue. 1994. « Writing scientific articles in English: solutions for French researchers? ». ASp 3, 56-64. 
Boughedaoui, Mourad. 1993. "Contribution à l'évaluation de l'aptitude des étudiants à interpréter les divers outils de cohésion dans le discours informatique écrit ». ASp 1, 107-128.

Boughedaoui, Mourad. 1997. « Contribution à l'amélioration de la compréhension et la traduction des adjectifs composés en classe de langue de spécialité ». ASp 15-18, 525-542.

Bruce, Nigel. 1999. «Classification and hierarchy in the discourse of wine: Émile Peynaud's 'The Taste of Wine'« . ASp 23-26, 149-164.

Brouzeng, Evelyne. 1993. «Approche interculturelle et enseignement des langues à des scientifiques. Rapide analyse linguistique ». ASp 1, 189-207.

Charpy, Jean-Pierre. 2011. « Les premiers dictionnaires médicaux en langue anglaise : glissements diachroniques du spécialisé au non spécialisé ». ASp 59, 25-42.

Corbisier, Agnès. 1993. « Enseigner à comprendre l'anglais de spécialité (informatique et gestion) 'par objectifs' et en 'visualisant l'information'« . ASp 1, 371-382.

Coulardeau, Jacques. 1995. « Computing English: the converging metaphors ». ASp 7-10, 41-56.

Crosnier, Elisabeth. 1994. « Enquête sur l'évaluation de l'anglais par les anglophones dans les publications des scientifiques français ». ASp 3, 39-55.

Dechet, Arlette. 1993. «Formation en anglais de spécialité dans les départements informatiques des IUT : choix des matériaux linguistiques et didactisation ». ASp 1, 139-188.

Dudley-Evans, Tony. 1993. « Subject specificity in ESP: How much does the teacher need to know of the subject?». ASp 1, 1-10.

English, Kathryn. 1998. « Understanding science: When metaphors become terms ». ASp 19-22, 151-164.

Fries-Verdeil, Marie-Hélène. 1998. « Les nombres dans la publicité scientifique ou technique : quand la langue de l'industrie devient l'industrie de la langue ». ASp 19-22, 121-134.

Fries-Verdeil, Marie-Hélène. 1999. « La vraie vie est ailleurs... fonction métonymique des réseaux dans quelques publicités britanniques pour le téléphone ». ASp 23-26, 165-176.

Fries-Verdeil, Marie-Hélène. 2001. «Les métaphores dans la publicité scientifique et technique : de la médiation à l'interaction ». ASp 31-33, 227-237.

Frost, Dan. 2004. « L'enseignement de l'accent lexical dans le secteur LANSAD : quelques notes sur une expérience de recherche-action en auto-apprentissage ». ASp 43-44, 99-106.

Frost, Dan. 2008. «The Stress Site : la conception d'un parcours multimédia pour un travail en autonomie sur l'accentuation des mots ». ASp 53-54, 111-127.

Genty, Stéphanie. 2009. « Apparent truth and false reality: Michael Crichton and the distancing of scientific discourse ». ASp 55, 95-106.

Humbley, John et Maria M. Negroni. 2004. « Termes techniques et marqueurs d'argumentation : pour débusquer l'argumentation cachée dans les articles de recherche ». ASp 45-46, 97-109.

Labrosse, Pierre. 2000. «Temps et aspect dans le commentaire des illustrations d'articles de recherche en informatique ». ASp 27-30, 155-184.

Laffont, Hélène, Susan Birch-Bécaas et Elisabeth Crosnier. 2003. « La vulgarisation du discours grammatical à l'intention des chercheurs spécialistes d'autres disciplines : transfert positif ou transfert négatif ? ». ASp 39-40, 143-160. 
Léonarduzzi, Laetitia. 1998. « What, where et how relatifs libres ou interrogatifs ? Une étude de l'anglais informatique ». ASp 19-22, 441-452.

Ludford-Lagrange, Catherine. 2000. « Inspired images and winning words in wine ads ». ASp 27-30, 517-526.

Magnet, Anne. 1999. « Réseaux de cohérence et de cohésion dans la pratique de l'anglais scientifique ». ASp 23-26, 85-98.

Magnet, Anne. 2000. «Stratégies de validation du discours scientifique : analyse diachronique du rôle de la section Discussion dans l'article de recherche ». ASp 27-30, 113-132.

Martin, Jacky. 1996. « Les enjeux du discours scientifique : la stratégie de véridiction ». ASp 11-14, 13-32.

Martin, Jacky. 1997. « Du bon usage des corpus dans la recherche sur le discours spécifique ». ASp 15-18, 75-84.

Martin, Jacky. 1998. «Science et rhétorique : la double articulation rhétorique du discours scientifique ». ASp 19-22, 5-16.

Mémet, Monique. 1993. «Enseignement de l'anglais de spécialité en génie électrique et informatique industrielle. Documents authentiques, niveaux de compétence professionnelle et niveaux de langue ». ASp 1, 575-591.

Mémet, Monique. 1995. « Content-based instruction in electrical engineering: collecting, organising and utilising data ». ASp 7-10, 407-420.

Miller, Thomas. 1993. "The introduction to the research article from a discourse perspective ». ASp 2, 55-68.

Peraldi, Sandrine, 2012. «L'anglais de spécialité en chimie organique : entre indétermination terminologique et multidimensionnalité ». ASp 62, 5-24.

Petit, Michel. 1997. «Stylistique(s) contrastive(s) du discours scientifique ». ASp 15-18, 139-156.

Pradeilles, Anne. 1993. « Niveaux d'énonciation, niveaux de culture en anglais informatique : l'incidence du niveau de culture professionnelle de l'apprenant sur la nature de la langue de spécialité à lui enseigner ». ASp 1, 11-34.

Salager-Meyer, Françoise. 1998. « Le discours aigre-doux de la controverse scientifique : évolution de la rhétorique des confrontations académiques ». ASp 19-22, 29-50.

Sionis, Claude. 1997. « The integration of verbal and non-verbal materials in some instances of written scientific discourse ». ASp 15-18, 339-356.

Sionis, Claude. 1997. « Written scientific discourse beyond words ». ASp 15-18, 357-372.

Sionis, Claude. 2000. « Inter-modal and inter-genre translation in scientific writing ». ASp 27-30, 185-198.

Williams, Geoffroy, Claude Sionis et Paul Boucher. 2002. « Single language corpus, multilingual background ». ASp 37-38, 47-57.

\section{Références}

Aguado De Cea Guadalupe \& Alejandro Curado Fuentes. 2012. « ESP in Spain: Goals, achievements and prospects ». ASp 62, 91-107.

Bazerman, Charles. 1988. Shaping Written Knowledge: The genre and the activity of the experimental article in science. Madison : University of Wisconsin Press. 
Bertin, Jean-Claude. 2008. «Le mot du président : unité et multiplicité du GERAS ». ASp « Les trente ans du GERAS », Numéro spécial, 2-6.

Biros, Camille. 2011. «Contribution à l'étude du discours environnemental : les organisations et leurs discours au Royaume-Uni ». Thèse de doctorat, Université Bordeaux Segalen.

Black, Max. 1962. « Metaphor ». Models and Metaphors. Ithaca New York : Cornell University Press, 24-49.

Black, Max. 1993 (2 éd. corrigée). « More about metaphor ». In Ortony A. (dir.), Metaphor and Thought. New York : Cambridge University Press, 19-41.

Cahiers de l'APLIUT 1 à 31, 1980- 2012. < http://apliut.com/> site consulté le 12/12/2012.

Chomsky, Noam. 1957. Syntactic structures. The Hague/Paris : Mouton.

Chomsky, Noam \& Morris Halle. 1968. The Sound Pattern of English. New York : Harper \& Row.

Dechet, Arlette, 1988. GOTO. L'anglais informatique pour communiquer. Paris : Ellipses.

Détourbe, Marie-Agnès. 2011. «Contribution à la caractérisation des pratiques d'évaluation dans le monde de l'enseignement supérieur britannique envisagé comme domaine spécialisé ». Thèse de doctorat, Université Bordeaux Segalen.

Détourbe, Marie-Agnès. 2012. «L'enseignement supérieur en contexte anglophone envisagé comme domaine spécialisé : aspects théoriques et méthodologiques ». ASp 61, 5-23.

English for Specific Purposes 1 à 31. 1980-2012. <http://journals.elsevier.com/> site consulté le $15 / 10 / 2012$.

Gallet-Blanchard, Liliane. 1990. L'anglais pour l'informatique. Paris : Dunod.

Ibérica 0 à 23, 1996-2012. <http://aelfe.org/> site consulté le 13/11/2012.

Laffont, Hélène. 2005. «Contribution à la caractérisation de l'anglais pour l'ingénieur ». Thèse de doctorat, Université Victor Segalen Bordeaux 2.

Lakoff, George. 1986. « The meaning of literal ». Metaphor and Symbolic Activity 1. Matwals, New Jersey : Lawrence Erlbaum Associates, 291-296.

Lakoff, George \& Mark Johnson. 1980. Metaphors We Live By. Chicago : University of Chicago Press.

Lakoff, George \& Mark Johnson. 1999. Philosophy in the Flesh, the embodied mind and its challenge to Western thought. Chicago : University of Chicago Press.

Leray, Yvette et alii. 1980. This Quiet revolution. Paris : A.Colin-Longman.

Mémet, Monique. 2001. «Thèses en anglais de spécialité ». In Mémet, Monique et Michel Petit (dir.). 2001. L'Anglais de spécialité en France, Mélanges en l'honneur de Michel Perrin. Bordeaux : GERAS-Éditeur Université Bordeaux 2, 344-352.

Mémet, Monique et Michel Petit (dir.). 2001. L’Anglais de spécialité en France, Mélanges en l'honneur de Michel Perrin. Bordeaux : GERAS-Éditeur Université Bordeaux 2.

Mémet, Monique et Michel Van Der Yeught. 2008. « Bref historique de ASp, la revue du GERAS ». ASp « Les trente ans du GERAS », Numéro spécial, 25-28.

Myers, Greg. 1990. Writing Biology. Texts in the Social Construction of Scientific Knowledge. Madison : University of Wisconsin Press.

Perrin, Michel. 1993. « Bulletin de naissance ». ASp 1, vii-ix. 
Petit, Michel. 1999. «La fiction à substrat professionnel : une autre voie d'accès à l'anglais de spécialité ». ASp 23-26, 57-81.

Petit, Michel. 2001. ASp 31-33. «Éditorial », iii.

Petit, Michel. 2002. La lettre du GERAS 22.

Petit, Michel et Shaeda Isani (dir.). 2004. Aspects de la fiction à substrat professionnel. Bordeaux : Presses universitaires de Bordeaux 2.

Statuts du Groupe d'étude et de recherche en anglais de spécialité, «Actes du V Colloque du GERAS, 20-21 Mars 1982 ». In Actes des $4^{e}$ et $5^{e}$ Colloques du GERAS. Bordeaux ; Montpellier : Universités de Bordeaux 2 et de Montpellier 3. Cahier 2, 45-47.

Swales, John M. 1990. Genre analysis. Cambridge : Cambridge University Press.

Trimble, Louis. 1985. English for Science and Technology. Cambridge : Cambridge University Press.

Trouillon, Jean-Louis. 2010. Approches de l'anglais de spécialité. Perpignan : Presses universitaires de Perpignan.

\section{NOTES}

1. ASp est la seule revue française consacrée exclusivement à la recherche en anglais de spécialité.

2. Voir le site du GERAS <http://www.geras.fr/>.

3. Les auteurs précisent que cette catégorie comprend « des articles qui traitent de l'analyse de la syntaxe, de l'analyse du discours, de stylistique, de phraséologie, d'analyse du genre, de lexicologie, de terminologie et de traduction » (2008:27-28).

4. Selon les auteurs, il s'agit là d'articles « qui traitent de théories et de pratiques, ainsi que de l'enseignement-apprentissage des langues de spécialité avec l'utilisation de technologies (télévision, vidéo, logiciels, laboratoires multimédia... » (2008:28).

5. Cette catégorie «comprend des articles traitant de la culture de domaines de spécialité (banque, publicité...) ; elle est proche de ce que les études anglaises nomment "civilisation » (2008: 28).

6. La liste des sources primaires est présentée en fin d'article, après la bibliographie.

7. Nous n'avons pas inclus dans notre corpus Mélanges en l'honneur de Michel Perrin (2001) car il s'agit là d'un ouvrage (classification ISBN) et non d'un numéro de la revue ASp (classification ISSN). Ne figure pas non plus dans cette étude le numéro spécial Les trente ans du GERAS, publié en 2008, qui est uniquement commémoratif.

8. Ce positionnement se veut donc plus englobant que celui qui a été adopté par M. Mémet et M. Van der Yeught (2008), dans lequel ils distinguent « informatique et technologie » d'une part, et « industries, sciences et techniques » d'autre part.

9. On pourra s'étonner que ne figure pas parmi les domaines de spécialité, l'œnologie qui apparaissait dans le tableau de J.-L. Trouillon (2010 : 50). L'article publié par Nigel Bruce (1999) analyse le discours sur le vin en tant que boisson pour gastronomes ; celui de Catherine LudfordLagrange (2000) porte sur l'analyse du discours publicitaire sur le vin. Ils n'entrent donc pas dans la catégorie retenue ici.

10. M. Van de Yeught (2012) opère la distinction entre les termes " approche » et " démarche » : «La démarche réalise [...] la synthèse des différentes analyses successivement développées par les approches linguistiques, discursives et culturelles et elle dessine le profil global de la LSP considérée ». 
11. Le terme "axe» avait initialement été proposé par Michel Perrin dans la demande d'habilitation du DEA de langue anglaise des spécialités scientifiques et techniques: méthodologie de la recherche linguistique, didactique, culturelle, technologique (1991). Il a été introduit au GERAS à partir du numéro 1 de la revue en 1993.

12. Cette expression est utilisée, dans la présente étude, pour nommer l'une des sous-catégories de l'approche didactique.

13. L'étude montre que ce type d'article tend progressivement à disparaître.

14. Nous avons inclus dans ces relevés les quelques articles écrits par des francophones, connus de nous, mais qui, pour des raisons diverses, avaient choisi de s'exprimer en anglais, par exemple Claude Sionis (1997 et 2000) ou M. Mémet (1995).

15. Parmi les ouvrages cités, certains sont des traductions. Nous les avons considérés dans la langue dans laquelle l'auteur les a consultés.

16. Le comité de lecture a été introduit en 1997 avec le $n^{\circ} 15-18$; jusqu'en 2000 (nº 27-30), la revue publiait aussi bien des articles acceptés par ledit comité que des articles de type compte rendu d'expérience ou encore de simples notes et fiches pédagogiques. Depuis 2001, année charnière, les articles sont publiés après acceptation par le comité de lecture constitué de deux relecteurs anonymes (blind reviewing).

17. Nous incluons dans ces établissements, les grandes écoles, les IUT, les universités et les classes préparant au BTS.

18. Deux auteurs francophones (J. Coulardeau, M. Mémet) ont rédigé en anglais.

19. Seuls Ann M. Johns et Geoffrey Leech font exception à cette règle.

20. Nous n'avons pas fait figurer dans les tableaux des auteurs très connus mais moins cités, comme Max Black (1962, 1993), George Lakoff $(1980,1986,1999)$ ou Mark Johnson $(1980,1999)$, surtout mentionnés dans les travaux de Marie-Hélène Fries (2001) sur la métaphore. Ces mêmes auteurs sont également cités par Kathryn English (1998) qui travaillait aussi sur la métaphore.

21. Notamment divers ouvrages d'apprentissage de l'anglais de l'informatique, This Quiet Revolution (Leray et al. 1980), Goto (Dechet 1988), L'Anglais pour l'informatique (Gallet-Blanchard 1990). Les autres disciplines ont été, à cette période, moins créatrices de manuels.

22. A. Magnet $(1999,2000)$.

23. Comme M.-H. Fries $(1998,1999,2001)$ et la métaphore, ou D. Banks $(2006,2009,2012)$ et la diachronie par exemple. Cette particularité explique la distribution dans le temps de certaines ressources bibliographiques, comme nous l'indiquons supra dans le cas des travaux de C. Bazerman et de G. Myers.

24. En 2009 D. Banks a publié deux articles.

25. Le numéro 4 de la revue ASp était intégralement consacré aux actes de l'atelier «New Technologies » du Congrès ESSE de 1993.

26. Les publications en lien avec l'enseignement sont bien plus nombreuses à cette même période (quinze en 1993 et sept en 1994). Voir tableau 7.

27. Le nombre de publications consacrées à l'enseignement est alors bien inférieur (7 en 1997 et 0 en 1998). Voir tableau 7.

28. Cette distinction apparaît dans la publication spéciale « Les trente ans du GERAS »: JeanClaude Bertin (2008) reprend l'article 2 des statuts du GERAS votés en mars 2007, dans lequel « anglais de spécialité » et "anglais pour spécialistes d'autres disciplines " sont clairement distingués. Dans la même publication, M. Mémet et M. Van der Yeught (2008) s'appuient eux aussi sur les statuts du GERAS, et dans cette version plus ancienne des textes (statuts votés en mars 1982) la distinction entre les deux secteurs n'apparaît pas.

29. En 2004, M. Petit dirige, avec la collaboration de Shaeda Isani, un ouvrage intitulé Aspects de la fiction à substrat professionnel, publié aux Presses universitaires de Bordeaux 2. 
30. Dans le cadre de l'étude que nous venons de mener, nous n'avons pas retenu la thématique de l'environnement que nous n'avons pas considérée comme appartenant exclusivement au domaine scientifique.

\section{RÉSUMÉS}

L'étude a pour objet de montrer comment les travaux menés par les chercheurs qui s'intéressent à l'anglais scientifique et qui ont publié leurs articles dans la revue ASp depuis 1993 reflètent l'influence du contexte de production. Par contexte de production, il faut entendre la politique universitaire des établissements d'enseignement supérieur, la place des chercheurs au sein de ces institutions ainsi que l'évolution de la recherche en anglais de spécialité au cours des vingt dernières années. Ces éléments peuvent expliquer les changements intervenus dans le choix des approches et des sujets retenus par les chercheurs. L'analyse de ces approches repose sur les axes historiques du GERAS qui sont la linguistique, la didactique, la culture et la technologie. Par les projections qu'elle permet d'envisager, cette étude rétrospective vise à éclairer la réflexion sur les évolutions futures de la recherche en anglais de spécialité, particulièrement en ce qui concerne l'anglais scientifique.

This study aims at showing how the work produced by researchers interested in scientific English and having published their papers in the journal ASp since 1993 reflects the influence of the general context within which they conducted their studies. By general context, we mean the political decisions that have influenced the organization and policy of higher education institutions, the researchers' role within these institutions and the evolution in ESP research throughout the last twenty years. Such elements may explain the changes in the subjects and approaches considered. The analysis of the approaches chosen by the researchers draws on the categorization around which the GERAS is organized, namely linguistics, didactics, culture and technology. This retrospective study thus aims at shedding light on the situation today and also at considering how research in the field of ESP and especially in scientific English could evolve in the coming years.

\section{INDEX}

Mots-clés : anglais scientifique, approche, ASp, contexte de production, évolution de la recherche

Keywords : approach, ASp, evolution of research, general context, scientific English

\section{AUTEURS}

\section{HÉLÈNE LAFFONT}

Hélène Laffont est maitre de conférences à l'INSA de Toulouse et membre de l'EA 4140. Sa recherche porte sur l'anglais de l'ingénieur et sur l'anglais du génie civil. Elle est le co-auteur, avec P. Bachschmidt, de l'ouvrage L'anglais de l'ingénieur, Guide pratique de la communication scientifique et technique (Ellipses 2009). helene.laffont@insa-toulouse.fr 


\section{JEAN-LOUIS TROUILLON}

Maintenant retraité, Jean-Louis Trouillon était maître de conférences HDR à l'Université de Perpignan et membre de l'EA 4140. Il est l'auteur de nombreux articles et didacticiels et a publié deux ouvrages, Dictionnaire anglais-français, français-anglais de géopolitique et relations internationales (2006) ainsi que Approches de l'anglais de spécialité (2010). jean-louis.trouillon@wanadoo.fr 